\title{
Flu channel drug resistance: a tale of two sites
}

\author{
Rafal M. Pielak ${ }^{1,2}$, James J. Chou ${ }^{1 凶}$ \\ ${ }^{1}$ Department of Biological Chemistry and Molecular Pharmacology, Harvard Medical School, Boston, MA 02115, USA \\ 2 Program in Biological and Biomedical Sciences, Harvard Medical School, Boston, MA 02115, USA \\ $\bowtie$ Correspondence: james_chou@hms.harvard.edu \\ Received December 18, 2009 Accepted January 18, 2010
}

\section{ABSTRACT}

The M2 proteins of influenza A and B virus, AM2 and BM2, respectively, are transmembrane proteins that oligomerize in the viral membrane to form proton-selective channels. Proton conductance of the M2 proteins is required for viral replication; it is believed to equilibrate $\mathrm{pH}$ across the viral membrane during cell entry and across the trans-Golgi membrane of infected cells during viral maturation. In addition to the role of $\mathrm{M} 2$ in proton conductance, recent mutagenesis and structural studies suggest that the cytoplasmic domains of the M2 proteins also play a role in recruiting the matrix proteins to the cell surface during virus budding. As viral ion channels of minimalist architecture, the membrane-embedded channel domain of M2 has been a model system for investigating the mechanism of proton conduction. Moreover, as a proven drug target for the treatment of influenza A infection, M2 has been the subject of intense research for developing new anti-flu therapeutics. AM2 is the target of two anti-influenza A drugs, amantadine and rimantadine, both belonging to the adamantane class of compounds. However, resistance of influenza $A$ to adamantane is now widespread due to mutations in the channel domain of AM2. This review summarizes the structure and function of both AM2 and BM2 channels, the mechanism of drug inhibition and drug resistance of AM2, as well as the development of new M2 inhibitors as potential anti-flu drugs.

KEYWORDS M2, BM2, influenza proton channel, amantadine, rimantadine

\section{INTRODUCTION}

In influenza virus $A$ and $B$, the $M 2$ proteins, referred here as $\mathrm{AM} 2$ and $\mathrm{BM} 2$, respectively, are single-span membrane proteins of around 100 residues, which tetramerize in the viral membrane to form $\mathrm{pH}$-dependent proton channels (Holsinger and Lamb, 1991; Sugrue and Hay, 1991; Paterson et al., 2003). It was first recognized in influenza $A$ that the role of the proton channel is to equilibrate $\mathrm{pH}$ across the viral membrane during entry and across the trans-Golgi membrane of infected cells during viral maturation (Hay et al., 1985; Helenius, 1992; Pinto et al., 1992). After endocytosis and before hemagglutinin (HA) mediated fusion between viral and endosomal membrane, the AM2 channels are activated by low $\mathrm{pH}$ of the endosome and conduct protons to acidify the viral interior. It has been suggested that the acidification weakens electrostatic interaction between matrix proteins and ribonucleoprotein (RNP) complexes such that subsequent membrane fusion can release the uncoated RNPs into the cytosol for transport into the nucleus (Martin and Helenius, 1991). This proposed mechanism, however, awaits further experimental validation. At a later stage during viral assembly, when newly synthesized viral proteins are transported to the cell surface by trans-Golgi network (TGN), the viral membrane proteins are topologically inverted in the TGN membrane such that the functional domain of $\mathrm{HA}$ is exposed to the low $\mathrm{pH}$ of the Golgi lumen and it is susceptible to premature rearrangement to the fusion-active conformation. In this case, the proton channels serve to conduct protons out of TGN to preserve the high $\mathrm{pH}$ form of nascent HA (Hay et al., 1985). Channel recording of AM2 in whole cells and reconstituted liposomes showed proton specific conductance that is activated by low pH (Pinto et al., 1992; Lin and Schroeder, 2001; Moffat et al., 2008; Pielak et al., 2009). The conductance of the wild type (WT) AM2 is inhibited by amantadine and rimantadine, which were the first effective drugs licensed for influenza treatment (Davies et al., 1964). The drug resistant variants of AM2 are, however, widespread, making these two drugs essentially useless today (Bright et al., 2006).

AM2 is a 97-residue single-pass membrane protein with its 
$\mathrm{N}$ and $\mathrm{C}$ terminus directed toward the outside and inside of the virus, respectively (Lamb et al., 1985). It consists of three segments: an extracellular $\mathrm{N}$-terminal segment (residues 1-23), a transmembrane (TM) segment (residues 24-46), and an intracellular C-terminal segment (residues 47-97). Crosslinking experiments showed that the channel is formed by a parallel tetrameric array of AM2 monomers, in which the formation of intermonomer disulfide bonds at Cys17 and Cys 19 may stabilize, but are not essential for, oligomeric assembly (Holsinger and Lamb, 1991; Sugrue and Hay, 1991). Mutagenesis studies have identified two pore-lining residues important for channel function; His37 is essential for channel activity and proton selectivity (Wang et al., 1995), and Trp41 is important for unidirectional conductance (Tang et al., 2002).

Multiple lines of evidences indicate that BM2, the 109residue $M 2$ protein from influenza $B$ virus, is a functional homolog of the AM2 proton channel. Similar to AM2, BM2 forms a homotetramer in the membrane (Paterson et al., 2003). After BM2 is synthesized in the infected host cell, it is incorporated into the TGN membrane and transported to cell surface for virus budding (Watanabe et al., 2003). BM2 proton conductance has a pH profile similar to that of AM2 (Mould et al., 2003). There are, however, significant differences between the two channels. BM2 channel activity is higher than that of AM2 (Mould et al., 2003; Wang et al., 2009), and, unlike AM2, the BM2 proton conductance is completely insensitive to amantadine and rimantadine (Mould et al., 2003). In fact, small molecule inhibitors that block the BM2 channel have not yet been identified. Although AM2 has been the subject of intense research due to the fear of influenza $A$ pandemic, BM2 has recently been gaining increased attention from the scientific community because influenza $B$ is a major constituent of human seasonal flu. According to Centers for Disease Control and Prevention (CDC), Influenza $B$ virus has caused infections that have totaled up to $50 \%$ of all influenza disease in recent years. Therefore, understanding how BM2 conducts protons and how it resists drug at the structural level is of considerable medical importance.

In addition to the membrane-embedded channel domain, AM2 and BM2 both have relatively large cytoplasmic regions compared to the other influenza surface proteins. The cytoplasmic regions of AM2 and BM2 have been suggested to play a role during viral assembly. Reverse genetics studies showed that deletions and mutations of the AM2 cytoplasmic region cause incomplete incorporation of genomic RNA into the virion and defective virus budding (McCown and Pekosz, 2006; Chen et al., 2008). These defects in viral assembly were attributed to disrupted association between the cytoplasmic region of AM2 and the M1 matrix protein. Similar experiments carried out on BM2 also suggest the important role of BM2 in virus assembly. Deletion of residues $51-80$ in the cytoplasmic region of BM2 dramatically reduced association of the matrix protein to the viral membrane and resulted in the failure of packaging of the RNP complex into the virion (Imai et al., 2004). Alanine-scanning mutagenesis of the region consisting of residues 86-109 also indicated involvement in virus assembly (Imai et al., 2008). Understanding the role of M2 cytoplasmic domains in virus assembly could offer new opportunities for developing anti-influenza therapeutics.

\section{STRUCTURES OF THE AM2 CHANNEL OF INFLUENZA A}

Obtaining a high resolution structure of the AM2 channel has been a long struggle because the small membrane protein resisted arduous efforts to crystallize, and it was not until about four years ago that solution NMR technology demonstrated the capability of tackling oligomeric channel-like membrane protein systems (Oxenoid and Chou, 2005). Before the availability of any high-resolution structures, a number of structural models of the channel region had been constructed by means of functional mutagenesis and computational modeling (Pinto et al., 1997), site-directed infrared dichroism (Kukol et al., 1999), and solid-state NMR (ssNMR) spectroscopy (Kovacs et al., 2000; Wang et al., 2001; Tian et al., 2003). Although the above models agree on the overall topology, they differ in the details essential for understanding the mechanism of channel opening, drug inhibition and drug resistance.

In 2008, using solution NMR spectroscopy, Schnell and Chou determined the first atomic resolution structure of the closed AM2 channel in the presence of the anti-influenza drug rimantadine (Schnell and Chou, 2008). One of the main challenges of solution NMR study of membrane proteins is finding a combination of protein construct and detergent system that both supports the native function of the protein and results in high quality NMR spectra. An AM2 construct including residues 18 to 60 , AM2(18-60), in dihexanoylphosphocholine (DHPC) detergent micelles met these requirements. This construct, which includes the TM domain as well as 15 residues of the C-terminal extension forms a very stable tetramer and yields high-quality NMR spectra (Schnell and Chou, 2008). It has also been confirmed that when reconstituted into liposomes, AM2(18-60) exhibits specific proton conductance that can be inhibited by as low as $10 \mu \mathrm{M}$ of rimantadine (Pielak et al., 2009). Consistent with the $\mathrm{pH}$ activated conductance in liposomes, $\mathrm{NMR} \mathrm{pH}$ titration experiments also showed dramatic changes in spectra as the $\mathrm{pH}$ of the sample was lowered, suggesting that the channels formed by AM2(18-60) in DHPC micelles could be opened by lowering $\mathrm{pH}$.

In the closed conformation ( $\mathrm{pH} 7.5), \mathrm{AM} 2(18-60)$ has an unstructured $\mathrm{N}$-terminus (residues 18-23), a channel-forming TM helix (residues 25-46), a short flexible loop (residues 47-50), and a C-terminal amphipathic (AP) helix (residues 51-59) (Fig. 1A). The TM helices assemble into a four-helix bundle with a left-handed twist angle of $\sim 23^{\circ}$ and a 


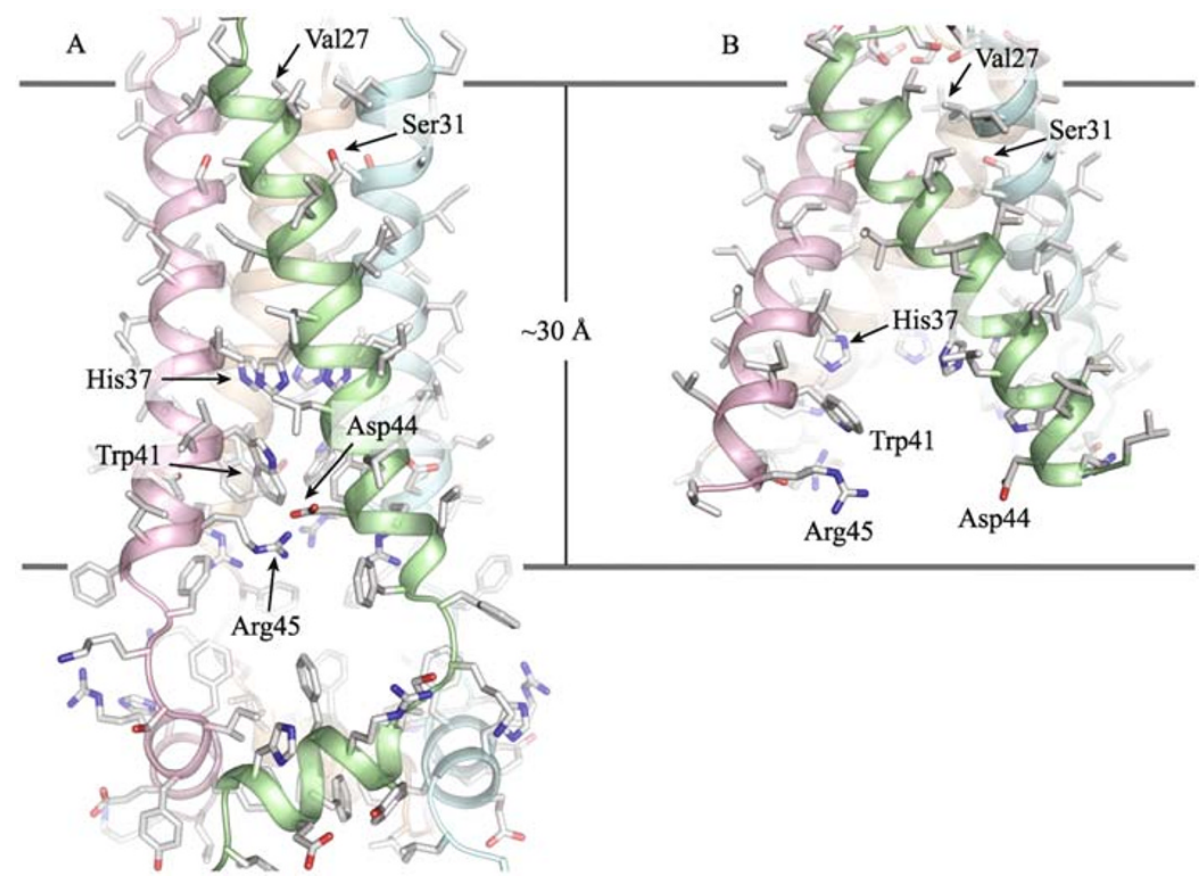

Figure 1. High resolution structures of the channel domain of AM2. (A) Solution structure in DHPC micelles at pH 7.5 (PDB code 2RLF). (B) Crystal structure in OG micelles at pH 7.3 (PDB code 3BKD).

well-defined pore. The N-terminal opening of the channel is very narrow ( $\sim 3 \AA$ in inner diameter), constricted by a ring of methyl groups from Val27. The pore gradually widens towards the C-terminal end, and becomes the widest at the Gly34 position with an inner diameter of $\sim 6 \AA$. In the $\mathrm{C}$-terminal half of the channel, the sidechains of His 37 and Trp41 form the narrowest points, too small to allow anything to pass. According to the 3-bond ${ }^{15} \mathrm{~N}-{ }^{13} \mathrm{C} \gamma$ scalar coupling constant $\left({ }^{3} \mathrm{~N}_{\mathrm{NC} \gamma}\right)$ of $1.5 \mathrm{~Hz}$, the His $37 \chi_{1}$ rotamer is predominantly trans, but experiences significant rotameric averaging. On the other hand, Trp41 is essentially locked in the closed channel, because ${ }^{3} J_{\mathrm{NC} \gamma}$ of $2.6 \mathrm{~Hz}$ indicates that the $\chi_{1}$ rotamer is locked at the trans conformation, and $\chi_{2}$ is also fixed at around $-120^{\circ}$ based on NMR nuclear Overhauser enhancement (NOE) and residual dipolar coupling (RDC) data. The restricted motion of Trp41 is partly due to close packing of the four indoles that occludes the C-terminal pore. More importantly, Trp41 indole amine of one subunit is within hydrogen bonding distance of the Asp44 carboxyl carbon of the adjacent subunit. Thus the two residues form a specific intermolecular contact that stabilizes the Trp41 in the closed conformation. In addition, the Arg45 sidechain is also in proximity of forming salt bridge that may further stabilize the closed state.

The C-terminal AP helix (residues 51-59) is roughly perpendicular $\left(\sim 82^{\circ}\right)$ to the TM helix, and its orientation and amphipathic character suggest that it lies on the surface of the membrane (Tian et al., 2003). The AP helices form a separate tetrameric domain with a head-to-tail assembly and a right-handed packing mode (Fig. 1A), which is connected to TM region by a short loop (residues 47-50). The function of the AP helix is to further stabilize the AM2 tetramer, in particular, when the TM helices open up during activation. The TM construct alone, without the AP helix, does not form a stable tetramer, which may explain the sample-to-sample variability in helix tilt observed by ssNMR (Li et al., 2007).

In a separate crystallographic study, DeGrado and coworkers determined the $2.05 \AA$ crystal structure of a shorter construct, AM2(22-46) with the I33M mutation, in $\beta$ octylglucoside (OG) detergent (Fig. 1B). Although the peptide was crystallized at $\mathrm{pH} 7.3$, which in principle supports the closed channel conformation, the crystal structure is significantly different from the closed channel structure determined by NMR. In the crystal structure, the four-helix bundle is tightly packed at the $\mathrm{N}$-terminal end of the TM domain as in the NMR structure, but the helices dramatically splay outward at an average angle of $\sim 40^{\circ}$ at the C-terminus. As a result of the large inter-helical angle, the $\mathrm{C}$-terminal end of the channel is wide open with no obvious structural features that support proton gating or selection. Moreover, in the same study, the authors reported another structure of AM2(22-46) with the G34A mutation, crystallized at pH 5.3 and in the presence of the drug amantadine. This structure is almost identical to that of the I33M mutant solved at pH 7.3 in the absence of drug. Therefore, given the available data, we cannot comment on whether the crystal structure in Fig. 1B corresponds to the open or the closed state. A plausible explanation for the striking difference between the NMR and the crystal 
structures, both experimentally determined to high resolution at a similar $\mathrm{pH}$, is the different lengths of constructs used in the two studies. The AM2(22-46) construct used in the crystallographic study does not contain the C-terminal region (residues 47-60) that was shown to be critical for a stable tetramer formation (Schnell and Chou, 2008) and native-like conductance (Ma et al., 2009).

\section{THE OPEN STATE OF THE AM2 CHANNEL}

It is widely recognized that the open or active state of the channel must support a hydrated pore for conducting protons because the only two polar residues above the Trp41, the hydrophilic residue at position 31 and the absolutely required His 37, are too far apart along the TM helix to relay protons in the absence of water molecules. Although a well-defined structure of the open channel is not yet available, some features of the open state may be gleaned from the solution NMR and X-ray studies. Channel activation occurs upon protonation of the His 37 imidazoles that are closely packed in the channel pore, which results in electrostatic repulsion that could substantially weaken helical packing in the TM domain. Presumably this widens the pore to admit water molecules. The NMR pH titration experiment showed that lowering the $\mathrm{pH}$ from 7.5 to 6.0 caused severe broadening of most of the TM NMR resonances due to exchange between multiple conformations (Schnell and Chou, 2008). Relaxationcompensated Carr-Purcell-Meiboom-Gill (CPMG) measurement (Loria et al., 1999) of millisecond timescale dynamics of the channel gate also supported a more dynamic view of the open state (Schnell and Chou, 2008). Comparison of the CPMG measurements of the Trp41 indole amine at different $\mathrm{pH}$ showed that as the $\mathrm{pH}$ was lowered from 7.5 to 6.0 , the chemical exchange rate increased by more than four-fold. The above NMR data suggest that the open state is not a unique structure like the closed state. AM2 lacks the extensive structural scaffolding observed in larger ion channels that undergo specific conformational changes between the open and closed conformation. Thus, AM2 appears to have evolved a two-state gating mechanism in which the closed state is structurally rigid, but the open state is dynamic with a loose quaternary structure.

The picture of multiple open channel conformers qualitatively agrees with the $2.05 \AA$ crystal structure of the TM peptide. In the absence of the AP helix, the TM helix does not assemble into a stable tetramer, and thus the crystal structure could represent an intermediate of channel opening despite being solved at high $\mathrm{pH}$ (7.3). An interesting feature of the crystal structure is that the packing of the tetramer deviates substantially from $\mathrm{C} 4$ rotational symmetry, i.e., the four pairs of helix-helix packing interfaces are significantly different from each other with some being more closely packed than others. The various helix-helix packing arrangements could partially represent the intermediate states of channel opening.
In contrast to the TM domain, the NMR resonances of the AP helices are essentially unaffected by lowering the $\mathrm{pH}$, indicating that the $\mathrm{C}$-terminal base of the tetramer remains intact and may be needed to preserve the overall tetrameric state of AM2 when the TM helices are destabilized in the open state. In addition to the AP helices, a pair of $\mathrm{N}$-terminal cysteines, Cys17 and Cys19, have been shown to form intermolecular disulfides in vivo (Holsinger and Lamb, 1991; Sugrue and Hay, 1991). Although these cysteines are not required for channel function, they are conserved in nature and may play a role in keeping the tetramer together in the open state.

\section{DRUG INHIBITION AND ONGOING CONTROVERSY}

The amantadine/rimantadine inhibition site is now a matter of intense debate. In fact, the mechanism of drug inhibition has been controversial for the past two decades. Before the availability of high-resolution structures, the drug-binding site has been predicted from the location of drug-resistance mutations. Mutations that confer amantadine/rimantadine resistance occur at many of the amino-acid sites including 26, 27, 30, 31, 34, and 38 (Hay et al., 1985; Wang et al., 1993). Most of these positions, including 27, 30, 31, and 34 , had been predicted to face the channel interior, which led to the hypothesis that the drugs bind inside of the channel. Many different drug binding sites in the channel pore have been proposed. Sansom and Kerr performed molecular modeling studies of amantadine binding and, by moving the drug along the channel lumen, found an energy minimum around positions 27 and 31 (Sansom and Kerr, 1993). They suggested that the hydrophobic adamantyl cage of the drug interacts with Val27 sidechains while the drug amino group forms favorable electrostatic interactions with the Ser31 hydroxyls (Fig. 2, pore site 1). Yi et al. also placed amantadine around residue 27 based on computer simulation, but the

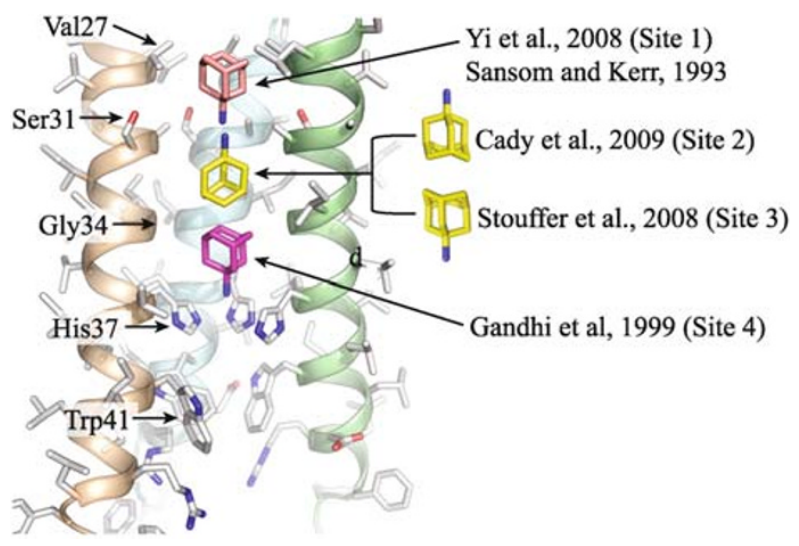

Figure 2. Where is the pore-binding site? This figure shows multiple amantadine pore binding sites proposed based on structural, functional, and/or computer simulation data. The protein structure shown here is the NMR structure of the closed channel (PDB code 2RLF). 
drug amino group formed one or more alternating hydrogen bonds between hydroxyls of Ser31 and backbone carbonyl of Ala30 (Fig. 2, pore site 1) (Yi et al., 2008). An experimental result consistent with the Val27 site is that of a neutron diffraction study, in which the authors determined that the drug is located $\sim 6 \AA$ from the center of the membrane (Duff et al., 1994). However, this experimental observation would also be consistent with a drug binding at Asp44 near the Cterminal end of the channel. Gandhi et al. recognized the importance of the His37 in proton conductance, and thus suggested that the amino group of amantadine may interact with imidazoles of His 37 whereas the hydrophobic cage of the drug is located in the widest region of the pore around Gly34 (Gandhi et al., 1999) (Fig. 2, pore site 4). However, this site would not be consistent with the above neutron diffraction data. Recently, Cady et al. suggested based on chemical shift changes measured by solid-state NMR that amantadine binds to yet another site inside the channel (Cady et al., 2009). In this binding site, the drug amino group points to the $\mathrm{N}$-terminal end of the channel and is located around Ser31 (Fig. 2, pore site 2). The authors noted, however, that the drug amine is too far from Ser31 hydroxyl groups $(\sim 4.5 \AA)$ to form hydrogen bonds (Cady et al., 2009). Although the experimental and modeling studies above all suggested interaction between amantadine and AM2, none of them directly showed the location of the binding site inside the pore. Interestingly, a recent mutagenesis study showed that mutating Ser31, which has been proposed to form polar contacts with the amino group of amantadine in pore sites 1 and 3 (Fig. 2), to alanine results in a drug sensitive mutant (Pielak et al., 2009), thus undermining some of the above proposed models.

While a pore binding site seemed to be a natural candidate in the absence of direct experimental evidence, its location awaits to be supported by "hard" evidence. The most direct evidence of a pore binding site came from a crystallographic study of the TM segment of AM2 peptide in the presence of amantadine (Stouffer et al., 2008). In this study an electron density on the axis of the pore adjacent to Ser31 was interpreted as a bound amantadine molecule (Fig. 2, pore site 3 and Fig. 3A). This interpretation, however, needs a higher resolution crystal structure in which the drug density assignment can be made more definitively. The crystal structure in the presence of amantadine was determined at relatively low resolution $(3.5 \AA)$, insufficient for unambiguous identification of small molecules such as amantadine (the maximum diameter of the roughly spherical adamantyl cage is $\sim 3.4 \AA$ ). The proposed model of drug binding in the pore features highly unusual pairing of hydrophobic and hydrophilic groups, wherein four hydroxyl groups of Ser31 coordinate the nonpolar adamantyl cage (Fig. 3B). An intriguing aspect of this binding mechanism is that the amantadine amino group, which has been shown by structure activity relationship (SAR) experiments to be critical for inhibition (Aldrich et al., 1971), does not appear to interact specifically with any of the polar groups within the channel pore. It is tempting to propose longrange polar interactions $(\sim 7 \AA$, Fig. 3B) between the drug amino group and the Ser31 hydroxyl groups; however, the S31A mutant is rimantadine sensitive (Pielak et al., 2009), indicating that Ser31 does not play a specific role in drug binding. Another remote possibility is that the drug binds nonspecifically inside the pore and that the crystal structure happened to capture one of the multiple binding states of the drug.

An alternative drug binding site has been observed by NOE experiments during structure determination of AM2(18-60) in detergent micelles. In this study, the authors observed a number of independent inter-molecular NOEs between rimantadine and a lipid-facing pocket at position 44 (Schnell and Chou, 2008) (Fig. 4A). There are in principle four equivalent binding sites accessible by the drug from the
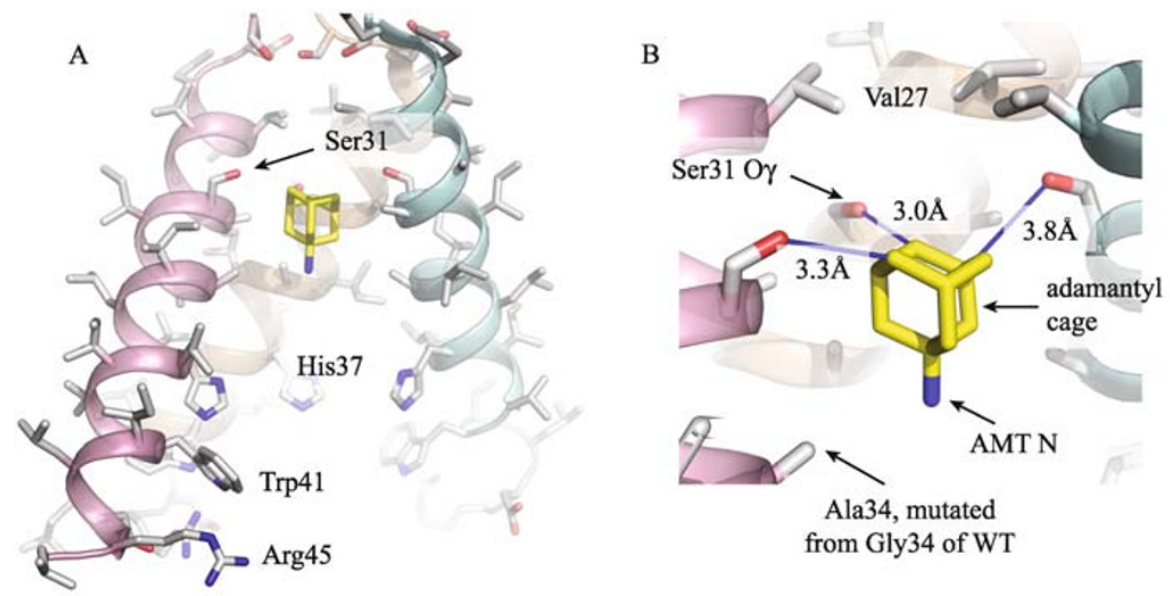

Figure 3. The amantadine binding site inside the pore of AM2 inferred by crystallographic density (PDB code 3C9J). (A) An overview of the drug binding location with respect to the channel. One of the four TM helices is hidden for clarity. (B) A detailed view of contacts between amantadine and residues in the channel pore. 


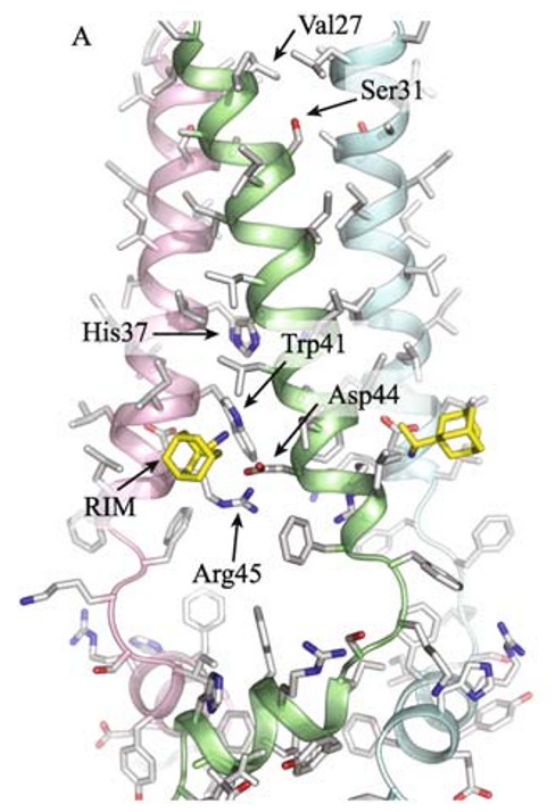

B

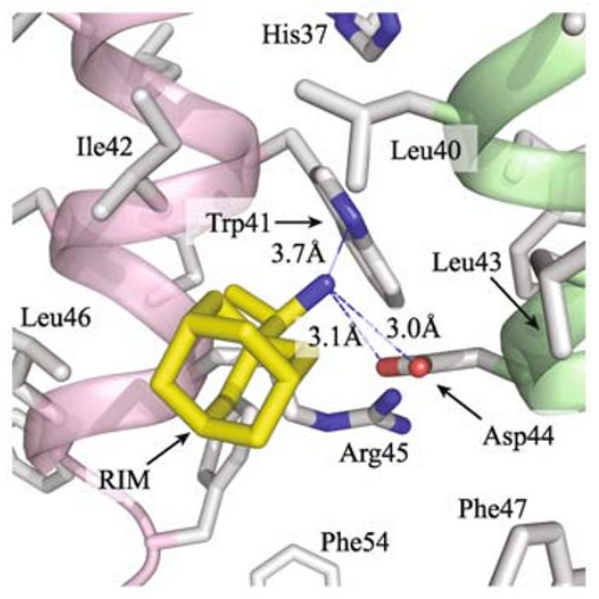

Figure 4. The lipid-facing rimantadine binding site of AM2 observed using solution NMR NOEs (PDB code 2RLF). (A) An overview of the drug binding location with respect to the channel. One of the four TM helices is hidden for clarity. Rimantadine (RIM) is colored in yellow. (B) A detailed view of contacts between rimantadine and residues in the lipid-facing pocket.

lipid bilayer, but the NMR study was not able to determine the binding stoichiometry. This lipid-facing pocket is formed by Trp41, Ile42, and Arg45 from one TM helix and Leu40, Leu43, and Asp44 from the adjacent TM helix, and shows a unique amphipathic property (Fig. 4B). In this pocket, the amino group of rimantadine is in contact with the polar sidechain of Asp44 and possibly also Arg45. The poly-cyclic hydrocarbon cage of the drug forms hydrophobic interactions with Ile42 from one TM helix and Leu40 and Leu43 from the adjacent helix. Although the relevance of the lipid-facing pocket to drug inhibition is still under heated debate, it is thus far the only drug binding site that has been unambiguously identified using direct protein-drug distance restraints. Since the drug binding pocket is composed of two adjacent helices, the drug binding inhibits the channel by stabilizing tight assembly of the closed conformation. Furthermore, the drug-binding site co-localizes with the predicted lateral proton exit at Asp44 (Schnell and Chou, 2008). Thus, in addition to stabilizing the closed conformation, the drug may directly block proton exit.

The inhibitory relevance of the lipid-facing site is supported by multiple mutagenesis, conductance, and structural experiments (Pielak et al., 2009). First, it has been shown using a liposomal proton flux assay that mutating Asp44, which forms polar contact with the amino group of rimantadine, to alanine completely abolishes drug binding and inhibition (Pielak et al., 2009). Second, mutating residues that form the hydrophobic walls of the pocket (Leu40, lle42, and Leu43) to alanines significantly reduces inhibition (Pielak et al., 2009). Third, inter-molecular NOEs between rimantadine and the lipidfacing pocket could no longer be detected in the drug resistant mutant $\mathrm{S} 31 \mathrm{~N}$, even though the experimental condition was identical to that used for measuring drug NOEs for the WT (Pielak et al., 2009). The above functional and structural studies suggested that the lipid-facing pocket observed in WT is neither an artifact of the technique used nor of the high drug concentration used for determining the structure; one would expect the same artifact for the S31N mutant. Fourth, the BM2 channel, which is resistant to amantadine, lacks the lipid-facing drug pocket (Wang et al., 2009), thus further supporting the relevance of this pocket. Finally, the lipid-facing pocket is supported by energetic analysis of the drug binding (Du et al., 2009).

There are, however, new studies reporting that a chimeric channel composed of the N-terminal TM segment of AM2 and the C-terminal segment of BM2 is sensitive to amantadine (Jing et al., 2008; Ohigashi et al., 2009). This result, obtained using the whole-cell channel recording technique, suggests that the site of drug action is located anywhere in the $\mathrm{N}$ terminal half of the TM region of the AM2-BM2 chimera. The result from the chimeric channel is completely inconsistent with the binding site observed by the NMR study (Schnell and Chou, 2008), which is supported by mutagenesis and liposome assay conductance measurement (Pielak et al., 2009). This inexplicable discrepancy awaits further resolution.

\section{MECHANISM OF DRUG RESISTANCE OF AM2}

While a mechanism of resistance based on a pore blocking model would be self-evident, a model of resistance to the 
allosteric inhibition is harder to explain. In this section we first present experimental evidences defining this mechanism.

Because the lipid-facing pocket is composed of residues from two adjacent TM helices, the stability and physical properties of the pocket must depend on the dynamics and conformation of helical packing. One would expect that changes either in inter-helical dynamics or in helix-helix packing would result in a change in drug binding affinity. Indeed, structural studies of the $\mathrm{S} 31 \mathrm{~N}$ resistant mutant, a mutant of the highly pathogenic avian influenza subtype H5N1, showed that replacing Ser31, which is located in the helix-helix interface, with the bulkier asparagine results in substantially weaker helix-helix packing (Pielak et al., 2009). Quantitative cross-linking experiments further support this observation. At a suitable concentration of dithiobis[succinimidylpropionate] (DSP) cross linkers, nearly all of WT could be cross-linked to a tetramer, whereas under the same conditions, the S31N mutant shows a distribution of monomer, dimer and tetramer on a denaturing gel (Pielak et al., 2009). Less efficient cross-linking indicates weaker tetrameric assembly for some regions of the protein; it does not suggest dissociation of the tetramer, because the mutants could all be cross-linked to nearly homogeneous tetramers under a stronger cross-linking condition (Pielak et al., 2009). Furthermore, the same NMR experiment used for observing proteindrug NOEs for the WT could no longer detect drug NOEs in the S31N mutant, suggesting that the mutation in the $\mathrm{N}$ terminal portion of the TM dramatically weakens drug binding to the lipid-facing pocket in the C-terminal TM region (Pielak et al., 2009). In addition to the S31N mutation, another prominent resistance mutation, V27A, also shows similar effect of destabilizing helix-helix packing (Pielak et al., 2009).

The above results suggest a model in which the mechanisms of drug inhibition and drug resistance of the AM2 channel are both allosteric. Drug binds to the tightly assembled pocket in the closed state, driving the equilibrium towards the non-conducting state. Resistance mutations such as S31N and V27A weaken TM helical packing and thereby disrupt the drug pocket. An intuitive picture for visualizing this mechanism is shown in Fig. 5. In this picture drug resistant mutants, such as S31N and V27A, assemble more dynamic, less defined, closed state. Proteins are dynamic entities that can be represented by continuous distribution of conformationally distinct states. In such a picture, WT closed state could be interpreted as a sharp Gaussian distribution of all possible closed conformations (Fig. 5, right panel). Drug resistant mutations, such as S31N or V27A, decrease the conformity of the closed state, which results in broadening of the Gaussian curve. Assuming that the drug binds only to the subset of conformations at the center of the Gaussian curve, flattening the distribution results in dramatic decrease of the closed states available for binding (Fig. 5, right panel).
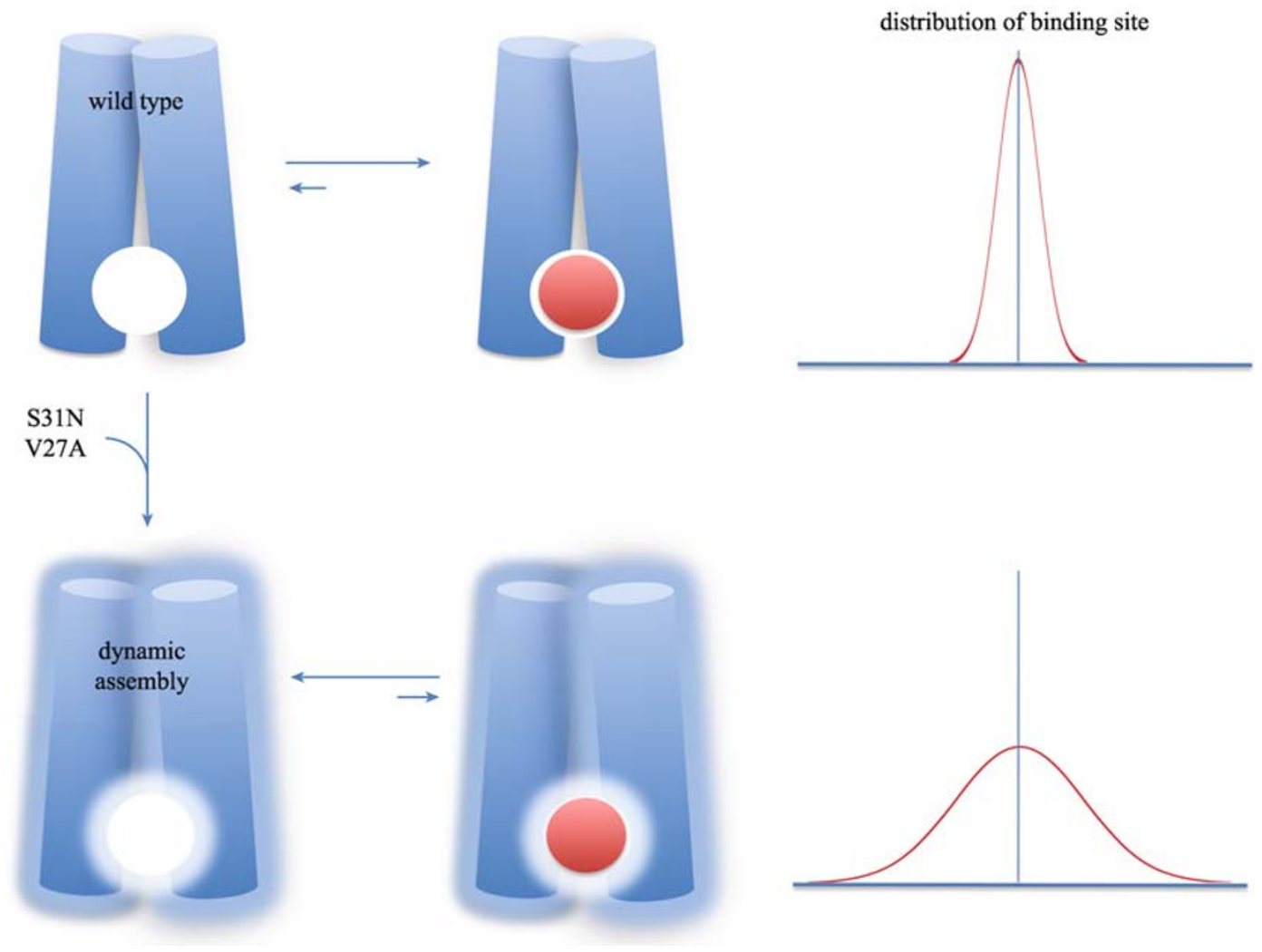

Figure 5. Allosteric mechanism of amantadine/rimantadine resistance of AM2. Drug is schematically represented in red. 
Why have resistance mutations closer to the external pocket not evolved? Residues 37-46 in the C-terminal portion of the TM domain, which includes residues forming the drug binding pocket, are highly conserved (Ito et al., 1991). This conservation is due to the functional constraints of His37, Trp41, Asp44, and Arg45 in gating, selectivity and proton relay. All other residues are leucines (positions 38, 40, 43 and 46) or isoleucines (positions 39 and 42) and are likely conserved to maintain membrane partitioning.

What would be the mechanism of resistance to the proposed drug binding inside the pore? While it is possible to explain resistance to any one of the proposed pore-binding sites in Fig. 2 conferred by single mutations, it is difficult to draw a mechanism that would account for all known resistance mutations. The resistance mutations, including L26F, V27A, A30T, S31N, G34E, and L38F, span more than three helical turns, but amantadine, which has a diameter of only $\sim 3.4 \AA$, cannot interact specifically with the entire $N$ terminal half of the channel. At pore site 1, the hydrophobic cage of amantadine interacts with Val27 sidechains and the amino group interacts with the hydroxyl groups of Ser31, and hence the V27A mutation could weaken binding at this position by decreasing hydrophobic contacts. At pore site 2, the adamantyl cage is surrounded by Gly34 and the amino group also interacts with Ser31 hydroxyls. The G34E mutation could interfere with drug binding at this position. For both of these binding sites, 1 and 2, the amino group of amantadine forms polar contacts with hydroxyl groups of Ser31. Thus mutation at Ser31 should dramatically decrease the effect of the drug. Indeed, S31N, the most prominent naturally occurring mutation confers drug resistance. However, an outstanding question remains: why does the S31A mutant, which does not at all support the above polar contacts, have a WT-like drug sensitivity (Pielak et al., 2009)? At pore site 4 , the amino group of amantadine interacts with His37 and the adamantyl cage is in vicinity of Gly34. This interaction could explain the G34E resistance mutation. However, unnatural mutants such as H37G and H37E remain drug sensitive (Wang et al., 1995). At pore site 3 , the drug adamantyl cage interacts with Ser31 and the drug amino group does not form any specific interactions. Therefore the S31N mutation could lead to steric interference due to the bulkier sidechain of asparagine. It is difficult to reason, however, why would the V27A mutation resist drug binding at site 3. Both NMR and X-ray structures show that Val27 sidechains narrowly constrict channel opening at the $\mathrm{N}$ terminal end, too small for the drug to enter. Replacing Val27 with smaller alanines would in principle widen the channel entrance and would thus facilitate rather than resist drug binding and inhibition.

Finally, can we explain all known resistance mutations based on just one pore-binding site? At this point, we cannot provide a convincing explanation but it should invoke some types of allosteric mechanism, such as the one proposed for the lipid-facing pocket.

\section{BM2 STRUCTURE AND FUNCTION}

Although BM2 (109 residues) is a functional homolog of AM2, the two proteins share almost no sequence homology except for the HXXXW sequence motif in the TM domain. Their domain sizes are also different. The $\mathrm{N}$-terminal unstructured segment preceding the TM domain of BM2 is very short (residues 1-4), whereas its C-terminal cytoplasmic domain is longer than that of AM2 by about 27 residues.

As in the case of AM2, establishing a suitable NMR system for structural studies of BM2 has been very challenging. In particular, it was difficult to find a detergent that preserves the structural integrity of both membrane-embedded and watersoluble domains. To determine the structure, Wang et al. established separate NMR systems for the TM and cytoplasmic domains (Wang et al., 2009). For the channel domain, they used the BM2(1-33) construct that has been shown by the liposome assay to have $\mathrm{pH}$ dependent proton conductance (Otomo et al., 2009; Wang et al., 2009). BM2(1-33) yields good NMR spectra when reconstituted in dihexonyl-

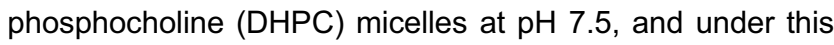
condition, the protein moves as a homogenous tetramer in SDS-PAGE without any chemical cross linking. For the cytoplasmic region, the construct BM2(26-109) was used, which includes the entire predicted extramembrane region (residues 44-109) as well as six N-terminal residues that overlap with BM2(1-33). Since the N-terminal part of BM2 (26-109) associates with membrane, solution NMR studies of this construct were carried out when the protein was anchored to LMPG (14:0 lyso phosphoglycerol) micelles. NMR structure determination of the above two constructs showed two structured domains of BM2, a TM domain in DHPC micelle that resembles the AM2 channel structure and a much larger cytoplasmic domain. Fig. 6A illustrates their localization relative to presumed lipid bilayer.

\section{Structure of the BM2 channel domain}

In DHPC micelles, BM2(1-33) forms a coiled-coil tetramer with a packing angle of about $-37^{\circ}$. The tetramer has a welldefined hydrophilic channel that is occluded by Phe5 and Trp23 at the $\mathrm{N}$-, and C-terminal ends, respectively (Fig. 6B).

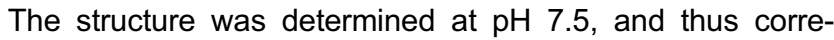
sponds to the closed state. Although the overall assembly of TM helices of BM2 is similar to that of AM2, e.g., both are lefthanded four-helix bundle having the key histadine and tryptophan in the pore, the two channels differ significantly in details. The TM domain of BM2 shows stronger coiled-coil characteristics with at least two heptad repeats: one from Leu8 at position $g$ to Ile14 at position $f$, and the other from Leu15 at position $g$ to lle21 at position $f$ (Fig. 6C). Positions a and $d$, which constitute the core of the coiled-coil tetramer, are occupied mostly by hydrophilic residues such as Ser9, Ser12, and Ser16. In addition to the serines, His 19 at position $d$ and Trp23 at position a are also pore-lining, consistent with their 

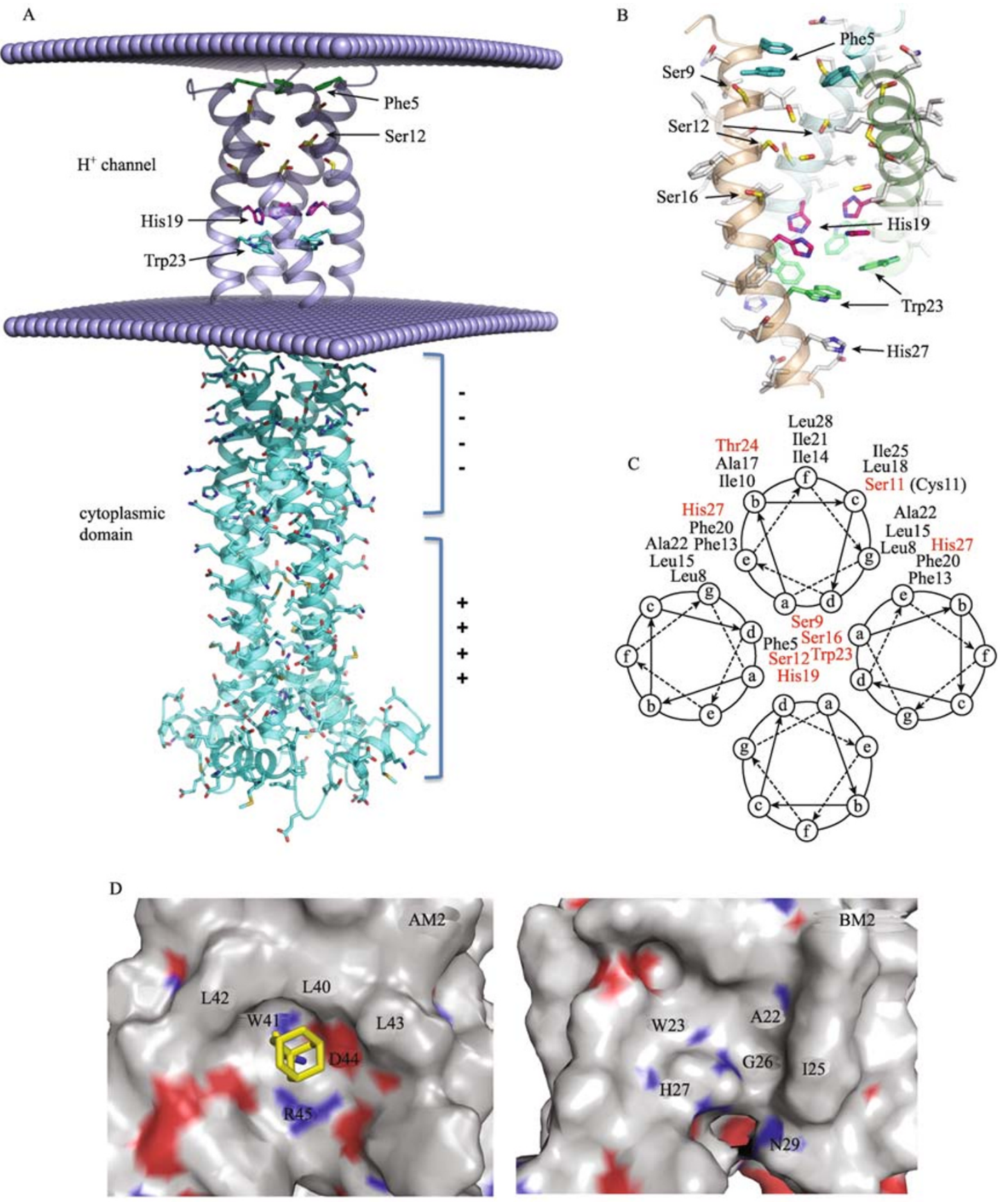

Figure 6. Structure of the BM2 protein. (A) The positioning of the two functional domains of BM2, the channel domain (PDB code $2 \mathrm{KIX}$ ) and the cytoplasmic domain (PDB code $2 \mathrm{KJ} 1$ ), and their localization relative to the presumed lipid bilayer. (B) The porelining residues in the channel domain revealed by removing one of the four TM helices. (C) Helical wheel representation of the BM2 channel assembly illustrates the concept of using the principle of coiled-coil packing to form a polar pore in a hydrophobic environment. Residues with negative or positive hydropathy index are colored in red and black, respectively. (D) BM2 channel domain does not have the rimantadine binding site observed in AM2. Left: the surface representation of the rimantadine-binding pocket in AM2 (PDB code 2RLF). Right: the surface representation of the corresponding region of the channel in BM2. 
essential roles in proton conductance and selectivity. Positions $g$ and $e$ are occupied by leucines 8 and 15 and phenylalanines 13 and 20 , respectively, to allow for peripheral hydrophobic interactions that stabilize helical packing. The rest of positions, $b, c$, and $f$, of the heptad repeat are occupied by hydrophobic residues (with the exception of Cys11), which form the hydrophobic surface of the tetramer for membrane partition. This arrangement for coiled-coil assembly in the membrane is the opposite to that of the water-soluble coiledcoil tetramer of GCN4, in which positions $a$ and $d$ are hydrophobic residues and positions $g$ and $e$ are polar residues (Harbury et al., 1993). The BM2(1-33) structure demonstrates the basic principles of forming a polar pore with coiled-coil assembly in the membrane environment. In fact, BM2 is the first of its kind in the known ion channel structures that adopts a coiled-coil assembly to conduct ions.

Compared to AM2, the BM2 channel pore has more polar groups than AM2, including serines 9, 12, and 16. Mutating the serines to alanine substantially reduced proton conductance, with the largest decrease $(\sim 45 \%)$ observed for S12A and S16A (Wang et al., 2009). These polar residues are important for hydration of the channel pore and for supporting proton passage to His 19. The conformation of the pore histidine with respect to the tryptophan gate in BM2 is the same as that in AM2. The structural conservation of the $\mathrm{pH}$ sensing histadine between AM2 and BM2 is consistent with its critical function in channel activation. The H27A and Q30A mutations on the C-terminal side of the Trp23 gate also reduced conductance by $\sim 25 \%$. These residues are exposed to the hydrophilic region of the lipid bilayer; and probably facilitate proton exit. It is interesting to note that polar residues after the tryptophan gate are also present in AM2 (Asp44 and Arg45), and replacing Asp44 to alanine results in three-fold reduction in conductance (Pielak et al., 2009). Overall the BM2 structure shows more polar residues throughout the predicted proton passage along the channel, which explains its higher conductance than AM2 (Wang et al., 2009).

Another interesting difference between the two channels relevant to drug resistance is that the proposed lipid-facing adamantane binding pocket in AM2 is absent in BM2, which may explain the drug resistance of the BM2 channel. This drug pocket, observed in the NMR structure of the AM2 channel, consists of Trp41, lle42, and Arg45 from one TM helix and Leu40, Leu43, and Asp44 from the adjacent TM helix. The corresponding residues in BM2 are Trp23, Thr24, and His27 from one TM helix and Ala22, lle26, and Gly26 from the adjacent TM helix (Fig. 6D). The above two groups of residues are uncorrelated and thus do not constitute surfaces of similar electrostatic and hydrophobic properties. On the other hand, the proposed amantadine binding site inside the pore of AM2, consisting of serine hydroxyl groups at residue position 31 , is not unique to AM2. The similar ring of serine hydroxyls is present at position 12 of BM2, having approximately the same distance from the tryptophan gate as Ser31 in AM2. This structural comparison shows that the lipid-facing pocket is specific to adamantane and AM2, and thus maybe relevant to channel inhibition.

\section{Structure and function of the BM2 cytoplasmic domain}

The structured region of the cytoplasmic sequence, residues $44-103$, is also a coiled coil tetramer (Fig. 6A). Residues 45-85 form an uninterrupted helix that oligomerizes into a lefthanded coiled-coil tetramer. A hairpin-like structure, consisting of residues 86-92, connects the coiled-coil structure to a short amphipathic helix that is roughly perpendicular to the coiled-coil helix. This short helix, residues 93-103, packs against the hairpin region of the adjacent subunit such that the hydrophobic sides of the amphipathic helix and hairpin are protected from the solvent. The capping of the $\mathrm{C}$-terminal end of a coiled-coil tetramer with amphipathic helices has been observed in other viruses, e.g., the phosphoprotein of Sendai virus (Tarbouriech et al., 2000).

A striking feature of the BM2 cytoplasmic domain is that the protein surface is densely populated with charged residues and that the positive and negative charges are strongly segregated, resulting in a large electrostatic dipole moment at neutral pH (Felder et al., 2007). NMR chemical shift perturbation experiments showed that the cytoplasmic domain interacts specifically with the M1 matrix protein, indicating specific molecular recognition between the two proteins. The perturbed region identified, from residues 84 to 108 , is consistent with known deletions and mutations of BM2 that affect virus assembly. Viruses with BM2 deletion of 101-109 contained dramatically reduced RNP complex (Imai et al., 2008). The C-terminal deletion also greatly affected membrane association of M1. Furthermore, alanine-scanning substitution of three consecutive residues showed that the 86-88A, 89-91A, 93-94A, and 95-97A mutants did not grow normally, and contained significantly reduced levels of M1 and NP (Imai et al., 2008). The data from structural and reverse genetics studies indicate that the interaction between the cytoplasmic regions of the proton channels and matrix proteins play an important role in viral assembly. During virus budding, the matrix proteins and RNPs must bind or coat the plasma membrane such that budding would result in a properly assembled virus. Therefore, the membrane patch that is destined to bud out from the host cell must contain specific sites for recruiting the matrix protein and RNP complexes. AM1 can directly bind to the viral membrane via electrostatic interaction (Ali et al., 2000). But membrane alone is clearly insufficient to warrant specificity, because charged membranes of other organelles are present in the cell. More specific interactions for recruiting the M1-RNP complexes to the budding site come from the cytoplasmic regions of the integral membrane proteins in the virus, including the short tails of glycoprotein HA and NA, as well as the large cytoplasmic domain of M2. 


\section{SUMMARY AND PERSPECTIVES}

Differences in sequence and structure of AM2 and BM2 demonstrate the remarkable ability of influenza virus to employ different structural solutions leading to the same function. Even though both proteins adopted a four-helix bundle architecture, wherein histidine imidazoles and tryptophan indoles, in concert, play a role in selectivity, gating, and directionality of proton flux, the detailed assembly of the four TM helices is very different. Whereas BM2 forms a bona fide coiled-coil tetramer spanning the entire TM region, AM2 is tightly packed only at the $\mathrm{N}$-terminal half of the channel and the helices slightly splay apart toward the C-terminus. This packing mode results in weaker tetramer, which requires the C-terminal AP helices for stable assembly. While the $\mathrm{N}$ terminal channel region of AM2 and BM2 share some structural similarity, the C-terminal domains are predicted to be substantially different; there is almost no sequence homology between the cytoplasmic regions of AM2 and BM2 and the BM2 cytoplasmic domain is about 25 residues larger than that of AM2.

The high rate of mutations in RNA viruses presents an enormous challenge to design drugs for inhibiting viral replication. Even though amantadine and rimantadine were proven to be effective drugs for treating influenza infections, most of the infuenza $A$ isolates are now adamantane resistant. Designing a pore-blocking drug presents an attractive prospect and there are examples of such molecules (for instance, tetrabutylammonium (TBA) that inhibits the KcsA K ${ }^{+}$channel (Zhou et al., 2001; Yohannan et al., 2007)); however, due to the low conservation in the M2 N-terminal segment of TM, designing a pore-blocking inhibitor may prove to be arduous. For example, the virus could introduce many mutations in the channel region from residues 26-38 without substantial loss of channel activity (Hay et al., 1985; Wang et al., 1993).

Development of more effective inhibitors for the experimentally determined lipid-facing pocket presents much brighter perspective. The pocket is composed of very conserved residues that are directly involved in the channel function. Since this drug-binding pocket is located right at the proposed proton exit site, the drug binding at this place directly stops proton current. Moreover, because the pocket is located between two adjacent helices and the opening requires loosening inter-helical packing, drug binding at the lipid-facing pocket stabilizes closed conformation of the channel. All known resistance mutations in the $\mathrm{N}$-terminal half of the channel have similar effect: either to destabilize the channel assembly to weaken drug binding or to drive equilibrium towards the open state. Therefore, resistance could in principle be overcome if there is a drug that binds much stronger to this site than amantadine or rimantadine, which would shift the equilibrium towards the closed conformation.
Although the progress in designing better adamantane based inhibitors of the AM2 channel has been stalled for many years, recent breakthroughs in obtaining high resolution structures of AM2 and BM2 have generated the much needed hope for better understanding of these proton channels and new promises for better channel inhibitors. After all, AM2 was once a proven drug target. We have many reasons to believe that the new developments in obtaining accurate picture of drug inhibition and drug resistance of the M2 channels will lead to development of more specific M2 inhibitors for treating influenza infections.

\section{ACKNOWLEDGEMENTS}

We thank Matthew Call for insightful discussion and critical reading of the manuscript. This work was supported by the NIH grant AI054520 (to JJC).

\section{ABBREVIATIONS}

AP, amphipathic; CPMG, Carr-Purcell-Meiboom-Gill; DHPC, dihexanoyl-phosphocholine; DSP, dithiobis[succinimidylpropionate]; HA, hemagglutinin; NOE, nuclear Overhauser enhancement; OG, octylglucoside; RDC, residual dipolar coupling; RNP, ribonucleoprotein; TBA, tetrabutylammonium; TGN, trans-Golgi network; TM, transmembrane

\section{REFERENCES}

Aldrich, P.E., Hermann, E.C., Meier, W.E., Paulshock, M., Prichard, W.W., Snyder, J.A., and Watts, J.C. (1971). Antiviral agents. 2. Structure-activity relationships of compounds related to 1adamantanamine. J Med Chem 14, 535-543.

Ali, A., Avalos, R.T., Ponimaskin, E., and Nayak, D.P. (2000). Influenza virus assembly: effect of influenza virus glycoproteins on the membrane association of M1 protein. J Virol 74, 8709-8719.

Bright, R.A., Shay, D.K., Shu, B., Cox, N.J., and Klimov, A.I. (2006). Adamantane resistance among influenza $A$ viruses isolated early during the 2005-2006 influenza season in the United States. Jama 295, 891-894.

Cady, S.D., Mishanina, T.V., and Hong, M. (2009). Structure of amantadine-bound $M 2$ transmembrane peptide of influenza $A$ in lipid bilayers from magic-angle-spinning solid-state NMR: the role of Ser31 in amantadine binding. J Mol Biol 385, 1127-1141.

Chen, B.J., Leser, G.P., Jackson, D., and Lamb, R.A. (2008). The influenza virus $M 2$ protein cytoplasmic tail interacts with the M1 protein and influences virus assembly at the site of virus budding. $J$ Virol 82, 10059-10070.

Davies, W.L., Grunert, R.R., Haff, R.F., McGahen, J.W., Neumayer, E. M., Paulshock, M., Watts, J.C., Wood, T.R., Hermann, E.C., and Hoffmann, C.E. (1964). Antiviral Activity of 1-Adamantanamine (Amantadine). Science 144, 862-863.

Du, Q.S., Huang, R.B., Wang, C.H., Li, X.M., and Chou, K.C. (2009). Energetic analysis of the two controversial drug binding sites of the M2 proton channel in influenza A virus. J Theor Biol 259, 159-164.

Duff, K.C., Gilchrist, P.J., Saxena, A.M., and Bradshaw, J.P. (1994). Neutron diffraction reveals the site of amantadine blockade in the 
influenza A M2 ion channel. Virology 202, 287-293.

Felder, C., Prilusky, J., Silman, I., and Sussman, J. (2007). A server and database for dipole moments of proteins. Nucleic Acids Research 35, special Web Servers Issue.

Gandhi, C.S., Shuck, K., Lear, J.D., Dieckmann, G.R., DeGrado, W. F., Lamb, R.A., and Pinto, L.H. (1999). Cu(II) inhibition of the proton translocation machinery of the influenza $A$ virus M2 protein. J Biol Chem 274, 5474-5482.

Harbury, P.B., Zhang, T., Kim, P.S., and Alber, T. (1993). A switch between two-, three-, and four-stranded coiled coils in GCN4 leucine zipper mutants. Science 262, 1401-1407.

Hay, A.J., Wolstenholme, A.J., Skehel, J.J., and Smith, M.H. (1985). The molecular basis of the specific anti-influenza action of amantadine. EMBO J 4, 3021-3024.

Helenius, A. (1992). Unpacking the incoming influenza virus. Cell 69, 577-578.

Holsinger, L.J., and Lamb, R.A. (1991). Influenza virus M2 integral membrane protein is a homotetramer stabilized by formation of disulfide bonds. Virology 183, 32-43.

Imai, M., Kawasaki, K., and Odagiri, T. (2008). Cytoplasmic domain of influenza B virus BM2 protein plays critical roles in production of infectious virus. J Virol 82, 728-739.

Imai, M., Watanabe, S., Ninomiya, A., Obuchi, M., and Odagiri, T. (2004). Influenza B virus BM2 protein is a crucial component for incorporation of viral ribonucleoprotein complex into virions during virus assembly. J Virol 78, 11007-11015.

Ito, T., Gorman, O.T., Kawaoka, Y., Bean, W.J., and Webster, R.G. (1991). Evolutionary analysis of the influenza A virus $M$ gene with comparison of the M1 and M2 proteins. J Virol 65, 5491-5498.

Jing, X., Ma, C., Ohigashi, Y., Oliveira, F.A., Jardetzky, T.S., Pinto, L. H., and Lamb, R.A. (2008). Functional studies indicate amantadine binds to the pore of the influenza A virus M2 proton-selective ion channel. Proc Natl Acad Sci U S A 105, 10967-10972.

Kovacs, F.A., Denny, J.K., Song, Z., Quine, J.R., and Cross, T.A. (2000). Helix tilt of the $M 2$ transmembrane peptide from influenza $A$ virus: an intrinsic property. J Mol Biol 295, 117-125.

Kukol, A., Adams, P.D., Rice, L.M., Brunger, A.T., and Arkin, T.I. (1999). Experimentally based orientational refinement of membrane protein models: A structure for the Influenza A M2 $\mathrm{H}+$ channel. J Mol Biol 286, 951-962.

Lamb, R.A., Zebedee, S.L., and Richardson, C.D. (1985). Influenza virus M2 protein is an integral membrane protein expressed on the infected-cell surface. Cell 40, 627-633.

Li, C., Qin, H., Gao, F.P., and Cross, T.A. (2007). Solid-state NMR characterization of conformational plasticity within the transmembrane domain of the influenza A M2 proton channel. Biochim Biophys Acta 1768, 3162-3170.

Lin, T.I., and Schroeder, C. (2001). Definitive assignment of proton selectivity and attoampere unitary current to the M2 ion channel protein of influenza A virus. J Virol 75, 3647-3656.

Loria, J.P., Rance, M., and Palmer, A.G., 3rd (1999). A relaxationcompensated carr-purcell-meiboom-gill sequence for characterizing chemical exchange by NMR spectroscopy. J Am Chem Soc 121, 2331-2332.

Ma, C., Polishchuk, A.L., Ohigashi, Y., Stouffer, A.L., Schon, A., Magavern, E., Jing, X., Lear, J.D., Freire, E., Lamb, R.A., et al. (2009). Identification of the functional core of the influenza $A$ virus
A/M2 proton-selective ion channel. Proc Natl Acad Sci U S A 106, 12283-12288.

Martin, K., and Helenius, A. (1991). Nuclear transport of influenza virus ribonucleoproteins: the viral matrix protein (M1) promotes export and inhibits import. Cell 67, 117-130.

McCown, M.F., and Pekosz, A. (2006). Distinct domains of the influenza a virus M2 protein cytoplasmic tail mediate binding to the M1 protein and facilitate infectious virus production. J Virol 80 , 8178-8189.

Moffat, J.C., Vijayvergiya, V., Gao, P.F., Cross, T.A., Woodbury, D.J., and Busath, D.D. (2008). Proton transport through influenza A virus M2 protein reconstituted in vesicles. Biophys J 94, 434-445.

Mould, J.A., Paterson, R.G., Takeda, M., Ohigashi, Y., Venkataraman, P., Lamb, R.A., and Pinto, L.H. (2003). Influenza B virus BM2 protein has ion channel activity that conducts protons across membranes. Dev Cell 5, 175-184.

Ohigashi, Y., Ma, C., Jing, X., Balannick, V., Pinto, L.H., and Lamb, R. A. (2009). An amantadine-sensitive chimeric BM2 ion channel of influenza $B$ virus has implications for the mechanism of drug inhibition. Proc Natl Acad Sci U S A 106, 18775-18779.

Otomo, K., Toyama, A., Miura, T., and Takeuchi, H. (2009). Interactions between histidine and tryptophan residues in the BM2 proton channel from influenza B virus. J Biochem 145, 543-554.

Oxenoid, K., and Chou, J.J. (2005). The structure of phospholamban pentamer reveals a channel-like architecture in membranes. Proc Natl Acad Sci U S A 102, 10870-10875.

Paterson, R.G., Takeda, M., Ohigashi, Y., Pinto, L.H., and Lamb, R.A. (2003). Influenza B virus BM2 protein is an oligomeric integral membrane protein expressed at the cell surface. Virology 306 , 7-17.

Pielak, R.M., Schnell, J.R., and Chou, J.J. (2009). Mechanism of drug inhibition and drug resistance of influenza A M2 channel. Proc Natl Acad Sci U S A 106, 7379-7384.

Pinto, L.H., Dieckmann, G.R., Gandhi, C.S., Papworth, C.G., Braman, J., Shaughnessy, M.A., Lear, J.D., Lamb, R.A., and DeGrado, W.F. (1997). A functionally defined model for the M2 proton channel of influenza A virus suggests a mechanism for its ion selectivity. Proc Natl Acad Sci U S A 94, 11301-11306.

Pinto, L.H., Holsinger, L.J., and Lamb, R.A. (1992). Influenza virus M2 protein has ion channel activity. Cell 69, 517-528.

Sansom, M.S., and Kerr, I.D. (1993). Influenza virus M2 protein: a molecular modelling study of the ion channel. Protein Eng 6, 65-74.

Schnell, J.R., and Chou, J.J. (2008). Structure and mechanism of the M2 proton channel of influenza A virus. Nature 451, 591-595.

Stouffer, A.L., Acharya, R., Salom, D., Levine, A.S., Di Costanzo, L., Soto, C.S., Tereshko, V., Nanda, V., Stayrook, S., and DeGrado, W. F. (2008). Structural basis for the function and inhibition of an influenza virus proton channel. Nature 451, 596-599.

Sugrue, R.J., and Hay, A.J. (1991). Structural characteristics of the M2 protein of influenza A viruses: evidence that it forms a tetrameric channel. Virology 180, 617-624.

Tang, Y., Zaitseva, F., Lamb, R.A., and Pinto, L.H. (2002). The gate of the influenza virus M2 proton channel is formed by a single tryptophan residue. J Biol Chem 277, 39880-39886.

Tarbouriech, N., Curran, J., Ruigrok, R.W., and Burmeister, W.P. 
(2000). Tetrameric coiled coil domain of Sendai virus phosphoprotein. Nat Struct Biol 7, 777-781.

Tian, C., Gao, P.F., Pinto, L.H., Lamb, R.A., and Cross, T.A. (2003). Initial structural and dynamic characterization of the M2 protein transmembrane and amphipathic helices in lipid bilayers. Protein Sci 12, 2597-2605.

Wang, C., Lamb, R.A., and Pinto, L.H. (1995). Activation of the M2 ion channel of influenza virus: a role for the transmembrane domain histidine residue. Biophys J 69, 1363-1371.

Wang, C., Takeuchi, K., Pinto, L.H., and Lamb, R.A. (1993). Ion channel activity of influenza $A$ virus $M 2$ protein: characterization of the amantadine block. J Virol 67, 5585-5594.

Wang, J., Kim, S., Kovacs, F., and Cross, T.A. (2001). Structure of the transmembrane region of the $\mathrm{M} 2$ protein $\mathrm{H}(+)$ channel. Protein Sci 10, 2241-2250.
Wang, J., Pielak, R.M., McClintock, M.A., and Chou, J.J. (2009). Solution structure and functional analysis of the influenza B proton channel. Nat Struct Mol Biol 16, 1267-1271.

Watanabe, S., Imai, M., Ohara, Y., and Odagiri, T. (2003). Influenza B virus $\mathrm{BM} 2$ protein is transported through the trans-Golgi network as an integral membrane protein. J Virol 77, 10630-10637.

Yi, M., Cross, T.A., and Zhou, H.X. (2008). A secondary gate as a mechanism for inhibition of the $\mathrm{m} 2$ proton channel by amantadine. J Phys Chem B 112, 7977-7979.

Yohannan, S., Hu, Y., and Zhou, Y. (2007). Crystallographic study of the tetrabutylammonium block to the KcsA K + channel. J Mol Biol 366, 806-814.

Zhou, M., Morais-Cabral, J.H., Mann, S., and MacKinnon, R. (2001). Potassium channel receptor site for the inactivation gate and quaternary amine inhibitors. Nature 411, 657-661. 